\title{
Animal-Assisted Intervention: A Promising Approach to Obesity Prevention for Youth With Autism Spectrum Disorder
}

\author{
Aviva Must ${ }^{1 *}$, Christina M. Mulé ${ }^{2,3}$, Deborah E. Linder ${ }^{4}$, Sean B. Cash ${ }^{5}$ and Sara C. Folta ${ }^{5}$ \\ ${ }^{1}$ Department of Public Health \& Community Medicine, Tufts University School of Medicine, Boston, MA, United States, \\ ${ }^{2}$ Division of Developmental and Behavioral Pediatrics, Department of Pediatrics, University of Rochester School of Medicine \\ and Dentistry, Rochester, NY, United States, ${ }^{3}$ Division of Developmental and Behavioral Pediatrics, Department of Pediatrics, \\ Tufts University School of Medicine, Boston, MA, United States, ${ }^{4}$ Tufts Institute for Human-Animal Interaction, Cummings \\ School of Veterinary Medicine at Tufts University, North Grafton, MA, United States, ${ }^{5}$ Friedman School of Nutrition Science \\ and Policy, Tufts University, Boston, MA, United States
}

Keywords: animal-assisted intervention, obesity, human-animal interaction, human-animal bond, autism spectrum disorder

\section{INTRODUCTION}

OPEN ACCESS

Edited by:

Mitsuaki Ohta,

Tokyo University of Agriculture, Japan

Reviewed by:

Kate Morrissey Stahl,

University of Georgia, United States

*Correspondence:

Aviva Must

aviva.must@tufts.edu

Specialty section:

This article was submitted to Veterinary Humanities and Social

Sciences,

a section of the journal

Frontiers in Veterinary Science

Received: 24 December 2020

Accepted: 23 February 2021

Published: 19 May 2021

Citation:

Must A, Mulé CM, Linder DE, Cash SB and Folta SC (2021) Animal-Assisted Intervention: A Promising Approach to Obesity Prevention for Youth With

Autism Spectrum Disorder.

Front. Vet. Sci. 8:646081.

doi: 10.3389/fvets.2021.646081
In this opinion, we describe the justification for including companion dogs without special training (hereafter "pet dog") within a family, as an animal-assisted approach to health promotion for children with autism spectrum disorder (ASD). ASD is a neurodevelopmental disorder that is characterized by persistent social impairments, verbal and non-verbal communication difficulties, and restrictive and repetitive patterns of behaviors, activities, and/or interests (1). A recent US CDC survey estimates 1 in $54(1.85 \%)$ 8-year-old US children have been diagnosed with ASD (2). With obesity prevention as the key example, we review obesity risk factors in this population and demonstrate how a pet dog would, with appropriate considerations, contribute constructively to address the family-centered behavioral targets that encourage energy balance while also promoting health and wellness for the pet dog. The family environment represents a key venue for promoting heart healthy lifestyle behavior and one that is well-positioned for leveraging the human-animal bond. Food intake, physical activity, and sedentary behavior have been identified as the key drivers of excess weight gain in children and adults and are relevant for obesity prevention in youth with ASD as well as canine weight management and overall quality of life. Nonetheless, core features and several autism-associated characteristics present additional challenges and consideration when promoting behavior change for youth with ASD.

\section{OBESITY IN CHILDREN WITH ASD}

The obesity epidemic has spared no population group, with a growing body of evidence that children and adolescents with ASD are at increased risk compared with their neurotypical peers $(3,4)$. Estimates from the National Survey of Children's Health (NSCH), a periodic nationally representative survey, indicate a $30-50 \%$ increased risk for obesity in children with ASD compared to their non-autistic counterparts $(5,6)$. The most recent NSCH (2016) estimated an obesity prevalence of $23 \%$ among children with ASD aged 10-17 years compared to $16 \%$ in children without ASD (7). Although addressing obesity is a pressing public health problem for all children, it is particularly so for children with ASD for whom secondary health issues that manifest in adulthood may limit their access to the least restrictive future living arrangement. 


\section{MODIFIABLE OBESITY RISK FACTORS IN CHILDREN WITH ASD}

Obesity occurs when energy intake exceeds energy expenditure, net of the energy needed for growth. Both food intake and expenditure can be adversely impacted in children with ASD. Core features of ASD are impairments in social communication, language, and related cognitive skills and behavioral and emotional regulation and the presence of restricted, repetitive behaviors (1).

Addressing excess weight in a child with ASD must be considered in the context of its broad impact on family life. Parents and caregivers have many competing priorities beyond those of raising typically developing children. Children with ASD may attend school outside of the district. That additional travel, along with regular appointments with a range of specialists represent a heavy burden on families (8). These substantial demands, as well as meeting the needs of siblings and maintaining a "normal" family life, understandably may result in families placing a lower priority on healthy eating and physical activity patterns than might otherwise be the case $(9,10)$.

\section{Animal-Assisted Interventions}

Maintaining a healthy weight is a challenge for many children and adolescents irrespective of the presence of a neurodevelopmental disorder. Few studies have directly examined the relationship between dog ownership and child obesity in the general population-and all are observational. One study of Australian children found a moderate significant protective effect of dog ownership on overweight for younger children but not older children (11). A second study from the UK found no association between dog ownership and child obesity cross-sectionally at age 7 or prospectively from age 7 to age 9 (12). Physical activity is the major pathway that would link dog ownership to obesity. A meta-analysis of dog ownership and adult obesity found that dog owners engage in significantly more walking and physical activity than non-owners. Although the presence of a pet dog in a household does not insure engagement, children in households with a dog are significantly more likely to meet physical activity guidelines (13). To date, there have been no randomized controlled intervention studies designed to assess the impact of dog ownership on child weight status or physical activity; the PLAYCE PAWS trial, launched in 2020, will directly test this hypothesis in a pilot exploratory study (14).

Prevention approaches that have been successful in typically developing children may not be similarly successful in youth with ASD. Nonetheless, inclusion of animals in obesity prevention efforts represents a promising approach that considers many of the unique features of ASD. Animals are increasingly a part of therapy with children with ASD with a positive impact on social and emotional functioning $(15,16)$. Dogs, in particular, provide relatively simple social and behavioral cues that children with ASD are better able to interpret (17).

AAI in children with ASD represents an emerging approach that has potential for effective obesity prevention. A systematic review and meta-analysis in 2018 of AAI in children with ASD provides a useful summary of studies to date that employed an experimental design (15). However, studies that incorporated dogs are quite limited and rarely include pet dogs in the home, favoring interventions with trained therapy or service dogs. Small effects on social interaction were identified in two dog-assisted play therapy pilot studies conducted in Hong Kong $(18,19)$. In all of the studies, the AAI was feasible and acceptable to participants. It should be noted that preventive obesity interventions require very large sample sizes to demonstrate significant results, given that obesity arises due to a small, but cumulative, positive energy balance. Demonstrating significant results in an AAI intervention for this population would likely require a sample size greater than would be feasible to enroll. Given mounting evidence that AAI can improve sustained focus, emotional stability, attitudes toward learning, and progress on individual goals, its utility for establishing and maintaining positive lifestyle behavior changes holds promise $(20,21)$.

\section{DISCUSSION}

\section{Potential Role for Pet Dogs in Promoting Healthy Behaviors}

Although much of the evidence on AAI among children with ASD reflects the use of registered therapy animals or service animals, pet dogs offer several significant potential advantages over therapy dogs. It is not always possible for families to access registered therapy animals and their handlers, and service dogs can come with significant expense. Furthermore, interventions that include pet dogs may be more sustainable and generalizable than those with a registered therapy dog since the pet dog, as a key intervention component, is not removed from the child's environment at the end of therapy. Importantly, pre-existing human-animal bonds may be further strengthened, and novelty is removed as a potential motivator as the pet dog is ever-present in the home.

Pet dogs can provide "in-house" vehicles and prompts for the promotion of physical activity and positive nutrition behaviors in a number of ways. For children who may avoid physical activity because of gross-motor skill deficits, a pet dog does not judge or grow impatient when a child evidences these challenges. Anxiety, a common co-morbidity of ASD, could be mitigated by a program emphasizing a pet dog's non-judgmental attitude toward the child. This is consistent with the observation that dogs have a calming effect on children with ASD (22). Similarly, introducing nutrition-related concepts through the lens of the dog's health may serve to increase knowledge and awareness in a less stressful manner because the emphasis is not directed at the child's problematic eating behaviors. Dogs may also provide an indirect approach to modeling healthy eating behaviors without stigma (e.g., it is possible to discuss how overeating and obesity can lead to diseases in dogs without inducing guilt or blame in children). Additionally, planning a shopping list to meet a pet dog's dietary needs (given that many healthy fruits and vegetables can be shared among children and dogs) may be an accessible entrée to learning to plan grocery shopping. Other areas of pet care may provide similarly useful models for selfcare. A social story around a pet dog going to a veterinarian for 
a check-up can help prepare a child for understanding a visit to a medical provider in a setting that is distinct from a child's own potential anxiety around experiences such as being touched by a stranger or receiving a vaccination (23). These areas also provide opportunity to strengthen the bond between the family and the pet dog, while improving the health and well-being of the pet dog by increasing canine health literacy among families and promoting physical activity and proper nutrition for all family members, human and canine alike.

\section{Assessing the Fit}

Several important issues must be considered for any AAI with pet dogs for children with ASD. One key consideration is the relationship between the pet dog and the child. Based on their known characteristics, some dogs may be better suited for this type of intervention than others, and it is important to know the individual animal, their sensitivities, and temperament. Certain breeds (e.g., golden retrievers, Labradors) are commonly trained to be service or therapy dogs; however, many other breeds can enjoy their role as a suitable companion or other helping animal. Family knowledge of the specific dogs and their needs in training is likely to be one of the most important predictors of success. For example, herding breeds that require extensive physical activity and mental enrichment may not be the best fit; however, many Pit Bulls are registered therapy dogs and provide enrichment for their families as well as enjoy the work themselves. Incorporating older dogs who are more mature or waiting to introduce AAI with a pet dog once the relationship within the family dynamic is fully established is also crucial for success. Pet allergies among family members is an additional consideration.

In the context of the family, the pet dog should be socially motivated, healthy, and have basic obedience training. The child should have at least a neutral relationship with the dog, if not a positive one, with no history of negative interactions including physical harm or threat of physical harm to the dog. Importantly, the child should possess some safety awareness both for themselves and for the dog. Viewing AAI as a partnership in which the pet dog benefits from and enjoys interaction just as much as the child and caregivers is critical to successful integration of a dog "with a job" into the family unit. Families who have not had a dog in their households previously should be realistic about the care requirements of a pet dog as well as the financial resources required. Costs include food and treats, clothing for harsh climates, grooming, walking/boarding, training if a younger dog, and veterinary expenses including preventive healthcare. The authors advise consultation with experts and careful consideration for the wellbeing and best fit for all family members, especially the pet dog, before adoption occurs. Although pet dogs can provide many benefits, they have substantial needs of their own, including daily care, attention, veterinary healthcare and associated costs. Additionally, given the demands of raising child with ASD, adding another dependent being may be burdensome. For all of these reasons, decisions to add a pet dog to a family unit should be carefully considered.

\section{Beyond Obesity}

The potential of an animal-assisted approach for building skills that positively impact health are numerous. These include, but are not limited to, aspects of self-care, establishing healthy routines, and sleep hygiene. For example, many individuals with ASD have limitations in their ability to function independently and require assistance to master self-care skills $(24,25)$. These deficits can include daily skills such as hygiene, food preparation, and other health-promoting behaviors; food purchasing skills are also an area of specific concern (26).

\section{Research Needs and Considerations}

Several avenues of future studies are suggested to realize the potential of AAI with pet dogs to promote healthy weight in children with ASD. Formative research is needed to investigate who and how best to deliver an AAI with pet dogs. Possibilities include parents and specialists already working with children with ASD, including applied behavioral analysts or occupational therapists. Formative research could provide insights into the existing relationships between children with ASD and their pet dogs, as well as how physical activity and nutrition promotion might be integrated within family life in households that include a pet dog. With greater understanding of the child with ASD and the pet-owning family, interventions to promote healthy behaviors and meet the particular needs of this population while ensuring safety and well-being of the canine participants can be designed and evaluated.

Guidelines for the design of psychosocial intervention research for individuals with ASD (27) are applicable to trials aimed at health promotion through use of the pet dog. This phased "model" is non-prescriptive but starts with formulation of a new intervention technique and single-case or small pilot designs. If initial feasibility and efficacy is established, the final phases call for randomized clinical trials and community effectiveness studies (27). Relevant short-term outcomes would include engagement, participation, and enjoyment; mediumterm outcomes would include improvements in physical activity and eating behaviors. Longer term outcomes would include decreased risk of obesity and obesity-related chronic diseases, cost benefits, and optimal opportunities for independent living.

Finally, in preparing this opinion we seek to start a dialog, and invite experts in a range of disciplines, including special education, adaptive physical education, developmental and behavioral pediatrics, occupational therapy, veterinary medicine, and nutrition to share their perspectives on the opportunity to involve pet dogs in promoting healthy weight and wellness for youth with ASD.

\section{AUTHOR CONTRIBUTIONS}

AM, CM, DL, SC, and SF: conceptualization, writing - original draft preparation, writing - review, and editing. All authors contributed to the article and approved the submitted version. 


\section{FUNDING}

This work was supported by the National Center for Advancing Translational Sciences, National Institutes of Health, Award Number UL1TR002544 and by the Tufts Initiative for Global Obesity Research (TIGOR). The content is solely the responsibility of the authors and does not necessarily represent the official views of the NIH or Tufts University.

\section{REFERENCES}

1. American Psychiatric Association. Diagnostic and Statistical Manual of Mental Disorders (5th ed). Arlington, VA: American Psychiatric Association (2013). doi: 10.1176/appi.books.9780890425596

2. Maenner MJ, Shaw KA, Baio J. Prevalence of autism spectrum disorder among children aged 8 years-autism and developmental disabilities monitoring network, 11 sites, United States, 2016. MMWR Surveill Summ. (2020) 69:1-12. doi: 10.15585/mmwr.ss6904al

3. Chen AY, Kim SE, Houtrow AJ, Newacheck PW. Prevalence of obesity among children with chronic conditions. Obesity. (2010) 18:210-3. doi: 10.1038/oby.2009.185

4. Curtin C, Anderson SE, Must A, Bandini L. The prevalence of obesity in children with autism: a secondary data analysis using nationally representative data from the national survey of children's health. BMC Pediatr. (2010) 10:11. doi: 10.1186/1471-2431-10-11

5. Healy S, Aigner CJ, Haegele JA. Prevalence of overweight and obesity among US youth with autism spectrum disorder. Autism. (2019) 23:104650. doi: 10.1177/1362361318791817

6. Must A, Eliasziw M, Phillips SM, Curtin C, Kral TVE, Segal M, et al. The effect of age on the prevalence of obesity among US youth with autism spectrum disorder. Childhood Obesity. (2017) 13:25-35. doi: 10.1089/chi.201 6.0079

7. Tybor DJ, Eliasziw M, Kral TVE, Segal M, Sherwood NE, Sikich L, et al. Parental concern regarding obesity in children with autism spectrum disorder in the United States: national survey of children's health 2016. Disabil Health J. (2019) 12:126-30. doi: 10.1016/j.dhjo.2018.09.004

8. Nealy CE, O'Hare L, Powers JD, Swick DC. The impact of autism spectrum disorders on the family: a qualitative study of mothers' perspectives. J Fam Soc Work. (2012) 15:187-201. doi: 10.1080/10522158.2012.675624

9. Must A, Curtin C, Bandini LG. Barriers to physical activity in children with autism spectrum disorders: relationship to physical activity and screen time. J Phys Activity Health. (2015) 12:529-34. doi: 10.1123/jpah.2013-0271

10. Obrusnikova I, Miccinello DL. Parent perceptions of factors influencing afterschool physical activity of children with autism spectrum disorders. Adapted Phys Activity Q. (2012) 29:63-80. doi: 10.1123/apaq.29.1.63

11. Timperio A, Salmon J, Chu B, Andrianopoulos N. Is dog ownership or dog walking associated with weight status in children and their parents? Health Promot J Aust. (2008) 19:60-3. doi: 10.1071/he08060

12. Westgarth C, Heron J, Ness AR, Bundred P, Gaskell RM, Coyne K, et al. Is childhood obesity influenced by dog ownership? No cross-sectional or longitudinal evidence. Obesity Facts. (2012) 5:833-44. doi: 10.1159/000345963

13. Christian H, Trapp G, Lauritsen C, Wright K, Giles-Corti B. Understanding the relationship between dog ownership and children's physical activity and sedentary behaviour. Pediatr Obes. (2013) 8:392-403. doi: 10.1111/j.2047-6310.2012.00113.x

14. Ng M, Wenden E, Lester L, Westgarth C, Christian H. A study protocol for a randomised controlled trial to evaluate the effectiveness of a dog-facilitated physical activity minimal intervention on young children's physical activity, health and development: the PLAYCE PAWS trial. BMC Public Health. (2021) 21:51. doi: 10.1186/s12889-020-10034-7

15. Charry-Sánchez JD, Pradilla I, Talero-Gutiérrez C. Effectiveness of animalassisted therapy in the pediatric population: systematic review and metaanalysis of controlled studies. J Dev Behav Pediatr. (2018) 39:58090. doi: $10.1097 / \mathrm{dbp} .0000000000000594$

\section{ACKNOWLEDGMENTS}

We are deeply grateful to our research assistants (Molly Daffner, Eli Halbreich, and Elise Mitchell) and stakeholder panel members (Candice Colon, Sandy Sullivan, Edward Sanabria, Debra Gibbs, Jeremy Nisbet, Jennifer Nisbet, and Terri Farrell) who have been engaged in this work with us and whose contributions have been invaluable.

16. O’Haire ME, Guérin NA, Kirkham AC. Animal-assisted intervention for trauma: a systematic literature review. Front Psychol. (2015) 6:1121. doi: 10.3389/fpsyg.2015.01121

17. Berry A, Borgi M, Francia N, Alleva E, Cirulli F. Use of assistance and therapy dogs for children with autism spectrum disorders: a critical review of the current evidence. J Alternative Complement Med. (2013) 19:7380. doi: $10.1089 / \mathrm{acm} .2011 .0835$

18. Fung SC. Effect of animal-assisted play therapy on facilitating social behavior for children with autism: a preliminary comparison study. Human Animal Interact Bull. (2014) 2:40-59.

19. Fung SC. An observational study on canine-assisted play therapy for children with autism: move towards the phrase of manualization and protocol development. Glob J Health Sci. (2017) 9:67. doi: 10.5539/gjhs.v9n7p67

20. Friedmann E, Son $H$. The human-companion animal bond: how humans benefit. Vet Clin North Am. (2009) 39:293326. doi: 10.1016/j.cvsm.2008.10.015

21. Christian H, Trapp G, Villanueva K, Zubrick SR, Koekemoer R, Giles-Corti B. Dog walking is associated with more outdoor play and independent mobility for children. Prev Med. (2014) 67:259-63. doi: 10.1016/j.ypmed.2014.08.002

22. Burrows KE, Adams CL, Millman ST. Factors affecting behavior and welfare of service dogs for children with autism spectrum disorder. J Appl Animal Welfare Sci. (2008) 11:42-62. doi: 10.1080/10888700701555550

23. Nelson TM, Sheller B, Friedman CS, Bernier R. Educational and therapeutic behavioral approaches to providing dental care for patients with autism spectrum disorder. Special Care Dentistry. (2015) 35:105-13. doi: 10.1111/scd.12101

24. Carothers DE, Taylor RL. How teachers and parents can work together to teach daily living skills to children with autism. Focus Autism Other Dev Disabl. (2004) 19:102-4. doi: 10.1177/10883576040190020501

25. Cavkaytar A, Pollard E. Effectiveness of parent and therapist collaboration program (PTCP) for teaching self-care and domestic skills to individuals with autism. Educ Train Dev Disabil. (2009) 44:381-95.

26. Morse TE, Schuster JW, Sandknop PA. Grocery shopping skills for persons with moderate to profound intellectual disabilitiies: a review of the literature. Educ Treat Children. (1996) 19:487-517.

27. Smith T, Scahill L, Dawson G, Guthrie D, Lord C, Odom S, et al. Designing research studies on psychosocial interventions in autism. J Autism Dev Disord. (2007) 37:354-66. doi: 10.1007/s10803-006-0173-3

Conflict of Interest: DL was a faculty advisor for a Pet Partner community partner group at Tufts University. The funders had no role in the design of the study; in the collection, analyses, or interpretation of data; in the writing of the manuscript, or in the decision to publish the results.

The remaining authors declare that the research was conducted in the absence of any commercial or financial relationships that could be construed as a potential conflict of interest.

Copyright (c) 2021 Must, Mulé, Linder, Cash and Folta. This is an open-access article distributed under the terms of the Creative Commons Attribution License (CC BY). The use, distribution or reproduction in other forums is permitted, provided the original author(s) and the copyright owner(s) are credited and that the original publication in this journal is cited, in accordance with accepted academic practice. No use, distribution or reproduction is permitted which does not comply with these terms. 